\title{
DISPERSIVE DISTORTIONS OF SYSTEM CHARACTERISTICS OF BROADBAND TRANSIONOSPHERIC RADIO CHANNELS
}

\author{
Dmitriy Ivanov ${ }^{1^{*}}$ Vladimir Ivanov $^{1}$ Natalya Ryabova ${ }^{1}$ Mariia $_{\text {Ryabova }}{ }^{2}$ Alexey Kislitsin $^{1}$ \\ Andrei Chernov ${ }^{1}$ Nikita Konkin ${ }^{1}$ \\ ${ }^{1}$ Volga State University of Technology, Russia \\ ${ }^{2}$ Bauman Moscow State Technical University, Moscow, Russia
}

Modern radio systems that use a transionospheric channel, are required signal bandwidth expansion. However, with a significant bandwidth expansion, there is a problem of dispersive distortions signals due to different phase speed of its spectral components. Evaluation of distortions is required comprehensive research of frequency dispersion for such radio channels. The aim of the research is to solve this problem. Formulated the general provisions of theory of frequency dispersion of phase shift in a medium. Formulas are obtained for components of dispersion through the approximation of a refractive index of a wave at a satellite-to-Earth link. Dependencies were obtained to evaluate dispersion parameters of various orders by the integral characteristics of electron density profile. Expressions were obtained for band coherence of wideband transionospheric channels and method of its evaluation, also we present the algorithm for constructing diagnostic maps of bands coherence for different regions by data of receiving stations of satellite navigation systems.

Key words: Transionospheric channel, Frequency phase dispersion, Dispersion distortions, Band coherence

\section{INTRODUCTION}

At present there is an actual problem of expansion of the radio frequency band in the space communication systems and radar the Earth from space. However, it causes dispersive distortions of system characteristics of transionospheric channels and, as a result, distortion of signals. Solving this problem is impossible without a comprehensive study the effect of frequency dispersion at a new position. Frequency dispersion is related to the frequency dependence of phase speed of propagation of a wave in the medium. Differences the phase speeds of the spectral components of signals can lead to disturbances of the phase-frequency characteristics of spectrum and signal distortion, up to complete its "destruction" [02, 05]. It is known that the dispersion is caused by the frequency dependence of the refractive index of a medium. Transionospheric propagation is possible at frequencies above the critical frequency of the ionosphere. Therefore transionospheric propagation theory has its own characteristics associated with this condition. They are manifested in the fact that the refractive index can be represented as form at the powers of the ratio of the plasma frequency to the frequency of propagation waves. The components of the refractive index are considered as approximations to the refractive index. In this regard, there is a need to study the contribution of each approximation at parameters of dispersion.

Impulse response is usually considered as system characteristic of transionospheric channel when dispersion distortions are evaluated. Currently, more attention is focused at characteristic, that called the instantaneous power delay profile - PDP, which is equal to the square of the envelope of impulse response. In [03, 09], were showed that the degree of distortion of PDP can be evaluated with the use of a channel parameter called the band coherence (BC). It characterizes frequency range, where dispersive distortions are ignored. It is necessary to get relation between the parameters of transionospheric channel and coherence band, as well as to develop an algorithm and software for getting maps of coherence bands with the use of data of satellite navigation systems GLONASS and GPS in order to monitoring the power of distortions of PDP of transionospheric channels.

The aim of this research is conduct comprehensive study of dispersive distortions of PDP of transionospheric channels and methods of their control by monitoring based on regional maps of coherence bands with the use of satellite navigation systems.

\section{GENERAL PROVISIONS OF FREQUENCY DISPERSION OF PHASE SHIFT OF WAVE AT TRANSIONOSPHERIC PROPAGATION}

The equivalence principle [04] lets us consider the problem of propagation radio signals with a frequency response of the form (1) instead of the problem of propagation of a wave packet in a medium:

$$
H(f)=H_{0}(f) \cdot \exp [-i \varphi(f)]
$$

where $H_{0}(f)$ - amplitude-frequency characteristic (AFC) of a channel, $\varphi(f)$ - phase-frequency characteristic (PFC) of a channel, which is equal to phase shift of a wave in a medium, $f$ - frequency of electromagnetic wave.

It is assumed that if an AFC of a channel is not a constant in his band, there is frequency dispersion of amplitude, 
and if a PFC depends nonlinearly on frequency, there is a frequency dispersion of phase in a channel .

In the case of the problem of dispersive distortions, we considered dispersion effect on impulse response of a channel, which has a bandwidth $B_{c h}$ from $f_{1}$ to $f_{2}$ :

$$
h(\tau)=\int_{f_{1}}^{f_{2}} H(f) \cdot \exp i f \tau \cdot d f
$$

Function $P(\tau)=|h(\tau)|^{2}$ is called the instantaneous power delay profile (PDP) by the International Classification. In a channel with limited bandwidth $B_{c h}$ and central frequency $\bar{f}=\left(f_{1}+f_{2}\right) / 2$ PFC can be represent in form:

$$
\begin{gathered}
\varphi(\omega)=\varphi(\bar{\omega})+\varphi_{\omega}^{\prime} \cdot \Omega+\varphi_{\omega}^{\prime \prime} \cdot \frac{\Omega^{2}}{2 !}+\varphi_{\omega}^{\prime \prime \prime} \cdot \frac{\Omega^{3}}{3 !}+\ldots \approx \\
\approx \varphi(\bar{f})+2 \pi \tau_{g_{\left.\right|_{0}}} \cdot(F)+\pi \Phi_{\left.\right|_{0}}(F)^{2}+\frac{\pi}{3} v_{\left.\right|_{0}}(F)^{3}, \quad F \in\left[-\frac{B_{c h}}{2}, \frac{B_{c h}}{2}\right]^{3)}
\end{gathered}
$$

In this case there is phase dispersion 1, 2, 3-rd order, where $\tau_{g_{\left.\right|_{0}}}, s_{\left.\right|_{0}}, v_{\left.\right|_{0}}$ parameters of dispersion; $F=f-\bar{f}$. Also, in addition to the PFC it makes sense to introduce the function, which is called dispersive characteristic (DC) of a channel. Its significance lies in the fact that it contains only phase dispersion parameters. Really:

$$
\tau_{g}(F)=\frac{d \varphi}{d \omega} \approx \tau_{g_{\left.\right|_{0}}}+s_{\left.\right|_{0}}(F)+\frac{v_{\left.\right|_{0}}}{2}(F)^{2}
$$

Let us analyze the expression for a refractive index and a phase shift in case of transionospheric propagation. Recall that in the equilibrium state, electrons oscillate in a plasma at a frequency, which is called plasma frequency. Transionospheric propagation is possible when wave frequency is higher than the plasma frequency of maximum of the ionosphere, i.e. $\left(\frac{\omega_{p}^{2}(z)}{\omega^{2}}\right)<1$

In this case, gyrofrequency can be ignored in the expression for the refractive index and we represent it in the form of a Taylor series in powers of the ratio :

$$
\begin{gathered}
n(z)=\sqrt{1-\left(\frac{\omega_{p}^{2}(z)}{\omega^{2}}\right)} \approx 1-\frac{1}{2}\left(\frac{f_{p}^{2}(z)}{f^{2}}\right)-\frac{1}{8}\left(\frac{f_{p}^{2}(z)}{f^{2}}\right)^{2}-\frac{1}{16}\left(\frac{f_{p}^{2}(z)}{f^{2}}\right)^{3} \ldots= \\
=1-\frac{1}{2} \cdot\left(\frac{k \cdot N}{f^{2}}\right)-\frac{1}{8} \cdot\left(\frac{k \cdot N}{f^{2}}\right)^{2}-\frac{1}{16} \cdot\left(\frac{k \cdot N}{f^{2}}\right)^{3} \ldots
\end{gathered}
$$

Then the phase shift in a medium will has the following form:

$$
\begin{gathered}
\varphi(\omega)=\frac{1}{c} \int_{s}[\omega \cdot n(\omega, z)] d z \approx \omega \int_{s} \frac{d z}{c}- \\
-[\frac{1}{f \frac{\pi k}{c} \int_{s} N_{e}(z) d z}+\frac{1}{\alpha^{3}} \overbrace{\frac{\pi k^{2}}{4 c} \int_{s} N_{e}^{2}(z) d z}^{\alpha_{2}}+\frac{1}{f^{5}} \overbrace{\frac{\pi k^{3}}{8 c} \int_{s} N_{e}^{3}(z) d z}^{\alpha_{3}}]= \\
=\omega \int_{s} \frac{d z}{c}-\left[\frac{\alpha_{1}}{f}+\frac{\alpha_{2}}{f^{3}}+\frac{\alpha_{3}}{f^{5}}\right]
\end{gathered}
$$

We can neglect some summands in the square brackets in the expression of phase (8), if phase shift much smaller than $2 \pi$ (for engineering use can be considered less than one).

Next, we define the first three derivatives of phase by frequency in a point, which are characteristics of frequency dispersion:

$$
\begin{aligned}
& \tau_{g}(f)=\frac{d \varphi}{d \omega}(f)=\frac{1}{2 \pi}\left[2 \pi \int_{s} \frac{d z}{c}+\frac{\alpha_{1}}{f^{2}}+3 \frac{\alpha_{2}}{f^{4}}+5 \frac{\alpha_{3}}{f^{6}}+\ldots\right] \\
& s(f)=\frac{d \tau_{g}}{d f}(f)=-\frac{1}{\pi}\left[\frac{\alpha_{1}}{f^{3}}+6 \frac{\alpha_{2}}{f^{5}}+15 \frac{\alpha_{3}}{f^{7}}+\ldots\right] \\
& v(f)=\frac{d^{2} \tau_{g}}{d f^{2}}(f)=\frac{3}{\pi}\left[\frac{\alpha_{1}}{f^{4}}+10 \frac{\alpha_{2}}{f^{6}}+35 \frac{\alpha_{3}}{f^{8}}+\ldots\right]
\end{aligned}
$$

At a fixed frequency $f=\bar{f}$ values: $\tau_{g}(\bar{f}) \quad s(\bar{f}) \quad v(\bar{f})$ are parameters of phase dispersion of a channel at this frequency.

\section{EVALUATE OF SIGNIFICANCE OF VARIOUS APPROXIMATIONS FOR DC TRANSIONOSPHERIC CHANNEL}

Each of formulas 9 - 11 represents the sum of various approximations. Their significance is determined primarily by the frequency value. To optimize the calculations it is important to evaluate the frequency range for which individual approximations are important. Obviously, the first summand in 9 is independent of frequency and therefore does not effect the distortion of wideband signals. The second summand will be significant at high frequencies. Influence of the third and fourth summands will be significant with approach to a critical frequency of the ionosphere.

We investigated the contribution of approximations by numerical method. To evaluate the integrals in coefficients $\alpha_{1}, \alpha_{2}, \alpha_{3}$ it is necessary to calculate integrals of the profile of the electron density, its square and cube. To do this, we used a well-established model NeQuick. The integrals of the profiles were calculated by rectangles method. In this case the region of the profile from 70 to $1000 \mathrm{~km}$ was divided into two parts from 70 to 500 and from $500 \mathrm{~km}$ to $1000 \mathrm{~km}$. Then the first part was divided by points into equal parts with step $10 \mathrm{~km}$, and the second part - with step $50 \mathrm{~km}$. We used the following expressions:

$$
\begin{aligned}
& \int_{s} N_{e}(z) d z=10^{4} \cdot \sum_{i=7}^{49} N_{e_{i}}+5 \cdot 10^{4} \sum_{i=50}^{59} N_{e_{i}} \\
& \int_{s} N_{e}{ }^{2}(z) d z=10^{4} \cdot \sum_{i=7}^{49}\left(N_{e_{i}}\right)^{2}+5 \cdot 10^{4} \sum_{i=50}^{59}\left(N_{e_{i}}\right)^{2} \\
& \int_{s} N_{e}^{3}(z) d z=10^{4} \cdot \sum_{i=7}^{49}\left(N_{e_{i}}\right)^{3}+5 \cdot 10^{4} \sum_{i=50}^{59}\left(N_{e_{i}}\right)^{3}
\end{aligned}
$$


Calculations showed that the value of the integrals in the coefficients will much vary at changing geophysical situation (time of day, season, solar activity). Therefore, the results of study of dependencies (9-11) will partials.

For general solutions that satisfy to requirements of engineering evaluations, we considered, dependences on relative frequencies, that are equal ratio of an absolute frequency to a critical frequency $f_{c r}$ of the ionosphere $\hat{f}=f / f_{c r}$. Indeed, by adopting a simplified two-parameter model of the ionosphere, in which the parameters satisfy to condition: $\int N_{e}(z) d z=N_{m} \cdot H_{1}$ (where $N_{m}$ - the density for the global maximum of profile, $H_{1}$ - effective thickness of the ionosphere), we can see that all ratios $\alpha_{1} / f^{2}, \alpha_{2} / f^{4}, \alpha_{3} / f^{6}$ depend on the relative frequency, because $k N_{m}=f_{c r}^{2}$ and $\int N_{e}^{2}(z) d z=N_{m}^{2} \cdot H_{2}$ and $\int N_{e}^{3}(z) d z=N_{m}^{3} \cdot H_{3}$ Hypothesis about the dispersion characteristics dependence on relative frequency was used to obtain a common solution. At first, significance of approximations for DC (9) was investigated with the use of the hypothesis. We used the comparison of exact and approximate solutions for dependences of DC on the relative frequency. The exact solution was obtained using the formula:

$$
\tau(\hat{f})=\frac{1}{c}\left(10^{4} \cdot \sum_{i=7}^{49} \frac{1}{\sqrt{1-\frac{1}{\hat{f}^{2}} \cdot \frac{f_{0 i}^{2}}{f_{c r}^{2}}}}+5 \cdot 10^{4} \cdot \sum_{i=50}^{59} \frac{1}{\sqrt{1-\frac{1}{\hat{f}^{2}} \cdot \frac{f_{0 i}^{2}}{f_{c r}^{2}}}}+z_{0}\right)
$$

where $f_{0}$ - plasma frequency; $z_{0}$ - height of the start of layer.

The first (high frequency approximation), second and third approximations were calculated using the formulas:

$$
\begin{aligned}
& \tau_{1}(\hat{f})=\frac{z_{s}}{c}+\frac{\alpha_{1}}{2 \pi \cdot \hat{f}^{2} \cdot f_{c r}^{2}} \\
& \tau_{2}(\hat{f})=\frac{z_{s}}{c}+\frac{1}{2 \pi}\left(\frac{\alpha_{1}}{\hat{f}^{2} \cdot f_{c r}^{2}}+\frac{\alpha_{2}}{\hat{f}^{4} \cdot f_{c r}^{4}}\right) \\
& \tau_{3}(\hat{f})=\frac{z_{s}}{c}+\frac{1}{2 \pi}\left(\frac{\alpha_{1}}{\hat{f}^{2} \cdot f_{c r}^{2}}+\frac{\alpha_{2}}{\hat{f}^{4} \cdot f_{c r}^{4}}+\frac{\alpha_{3}}{\hat{f}^{6} \cdot f_{c r}^{6}}\right)
\end{aligned}
$$

where $z_{s}$ - distance from the Earth to the spacecraft.

The results of calculations of dependences of DC on the relative frequency for exact and approximate solutions are shown at Figure 2.

Analysis of the data showed that the first approximation is valid at $\hat{f}>6$, the second $6>\hat{f}>4,5$ and the third $4,5>\hat{f}>3$.

\section{DISTORTIONS OF PDP OF TRANSIONOSPHERIC CHANNELS}

Typically, a channel is set in the frequency domain through frequency characteristic. Jump to impulse response is implemented using the Fourier transform.

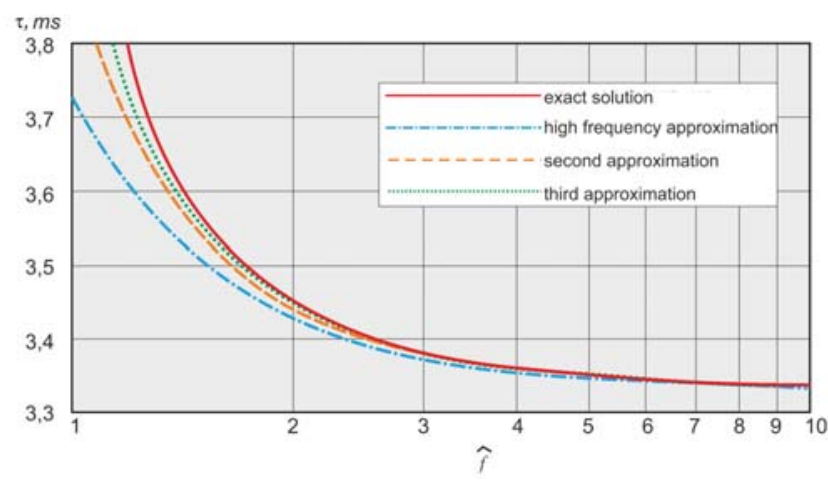

Figure 2: Dependencies of DC for exact solution and different approximations

In this case, it is assumed that there is no amplitude dispersion $\left(H_{0}(f) \approx H_{0}(\bar{f})\right.$ ) in a channel and phase dispersion is described using formula 8.

Solution for $P D P=P(\tau)$ is described by function $\sin c X$ [07] in the case of first-order dispersion, the Fresnel integrals [01] in the case of the second-order dispersion and for the third-order dispersion - incomplete Airy integrals [05, 08].

Numerical calculations lead to the form PDP, shown in Figure 3 , where it is assumed that, and maximum value is $\tau_{g}(\bar{f})=0$ and maximum value is $P D P=P_{m}(\tau)$. Note that the third-order dispersion leads to a peak at $v_{0}>0$ on the front.

PDP expands with an increase the parameter of second-order dispersion, and its peak power falls.

Peak appears at front edge of PDP, with increase the value of the third-order dispersion $v_{0}>0$. Changing the peak power is more complex than in the previous case.

\section{EVALUATION OF PDP DISTORTIONS BASED ON DATA OF COHERENCE BAND}

Nonlinear component of FRC distorts PDP [02, 08]. According to (3), there are linear and nonlinear components in the FRC formula:

$$
\varphi(\bar{f}, F)=\varphi_{l}(\bar{f}, F)+\varphi_{n l}(\bar{f}, F)
$$

where $\varphi_{l}(\bar{f}, F)=\varphi(f)+2 \pi \tau_{g_{\mid 0}}(F)$ - linear part of the phase relatively to frequency;

$\varphi_{n l}(\bar{f}, F)=\pi s_{\mid 0}(F)^{2}+\frac{\pi}{3} v_{\mid 0}(F)^{3}-$ nonlinear part of FRC.

If the third-order dispersion can be neglected, then nonlinear part of the FRC is a parabolic dependence on the frequency: $\quad \varphi_{n l}(f)=\pi s_{\mid 0}(F)^{2}$. Figure 6 shows form of FRC under different parameters of the second-order dispersion. 


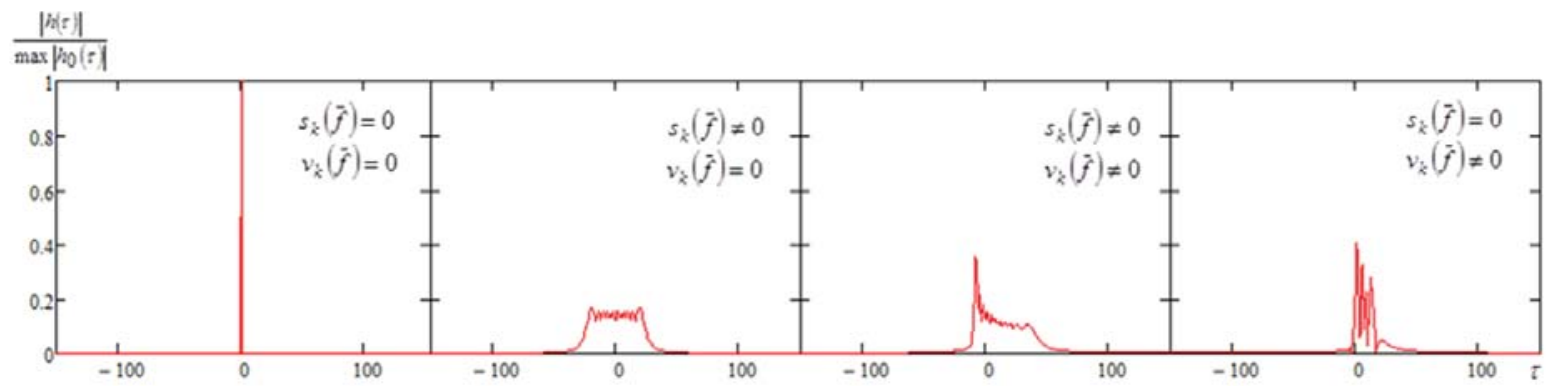

Figure 3: PDP of channels with the first-, second- and third-order dispersion , respectively

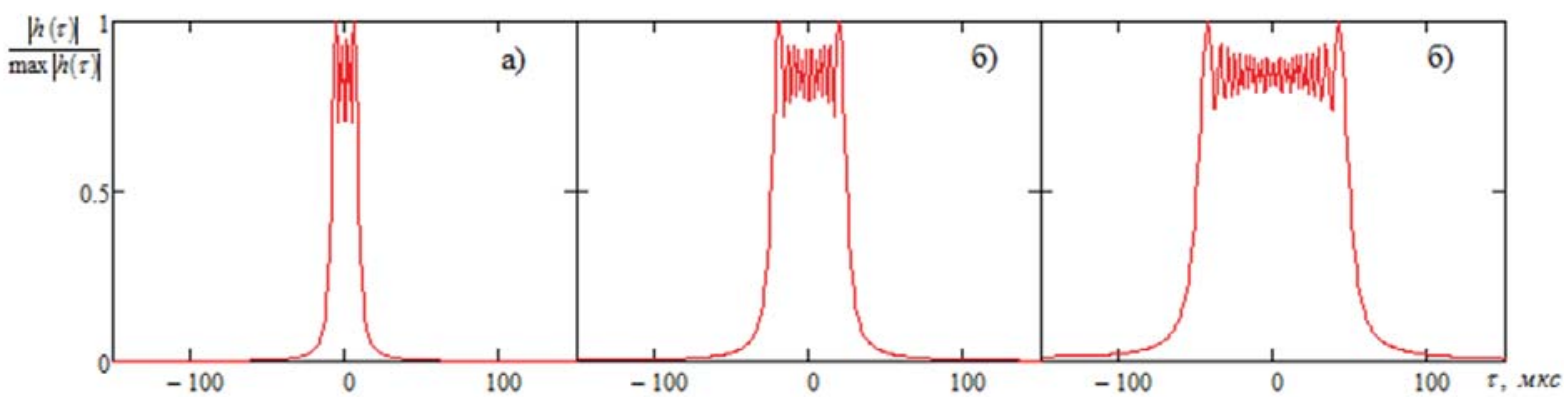

Figure 4: The increasing role of second-order dispersion with an increase of its value $s_{1}<s_{2}<s_{3}$

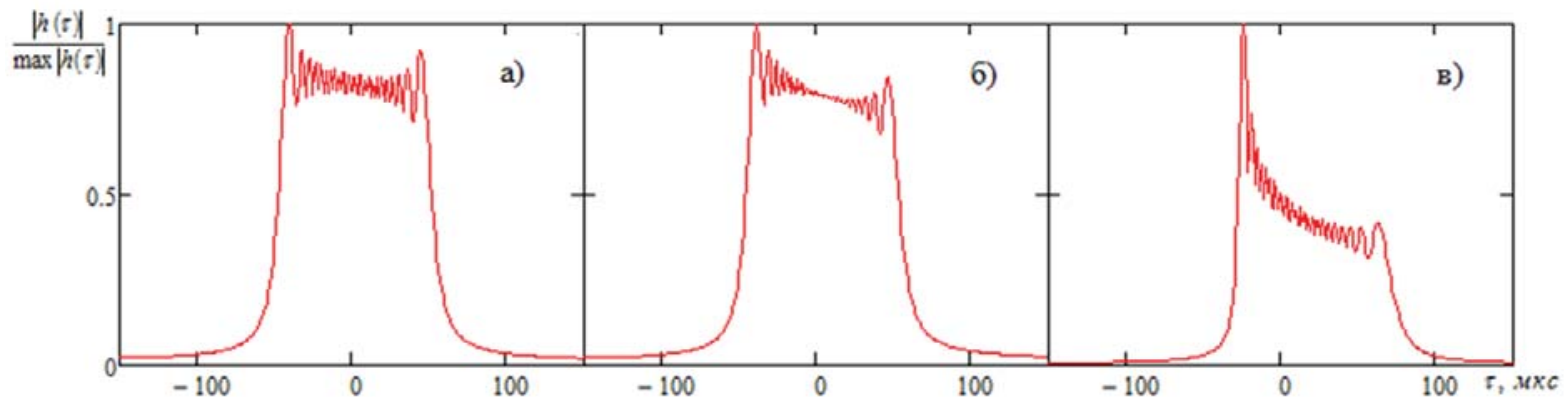

Figure 5: The increasing role of third-order dispersion with an increase of its value $v_{1}<v_{2}<v_{3}$

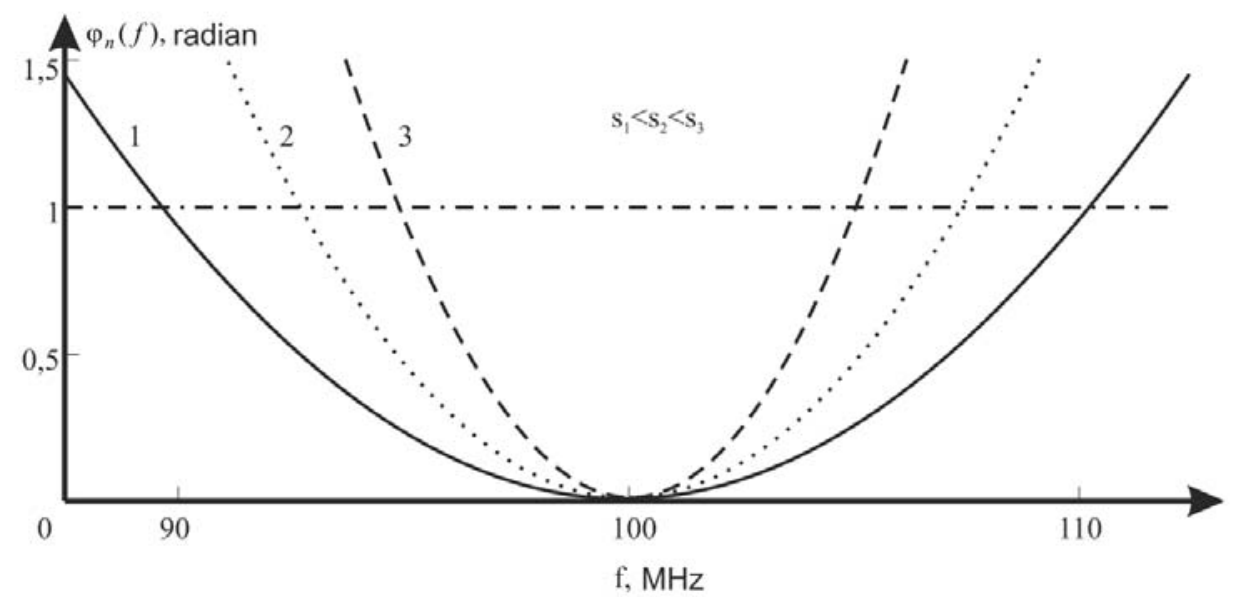

Figure 6: Form of nonlinear FRC of a channel for different slopes $s$ (1 - $30 \mu \mathrm{s} / \mathrm{MHz} ; 2$ - $60 \mu \mathrm{s} / \mathrm{MHz} ; 3$ - $120 \mu \mathrm{s} / \mathrm{MHz}$ ) 
The concept of coherence band is introduced to evaluate the significance of dispersive distortions. It is a band that has phase shift less than 1 radian on edges of its range (look at Figure 6), i.e. $\pi s_{0}(f-\bar{f})^{2}=1$, because at some frequencies which is on the left and right of a middle frequency of a channel there is violation of phasing of spectral components of a signal. Common view of the expression of the coherence band in the case of second-order dispersion is:

$$
B_{k}=\frac{2}{\sqrt{\pi \cdot \mid s} \mid}
$$

For transionospheric channel there is the following formula for determining the coherence band in the case of the HF approximation:

$$
B_{k}=\sqrt{\left(\frac{c \cdot f_{c r}^{3}}{k \cdot N_{t}}\right) \cdot \hat{f}^{3}}
$$

Figure 7 shows results of computing CB for the day and night time. The common solution for the frequency dependence of the $\mathrm{CB}$, obtained as the average of the data for the day and night time, is illustrated with a bold line.

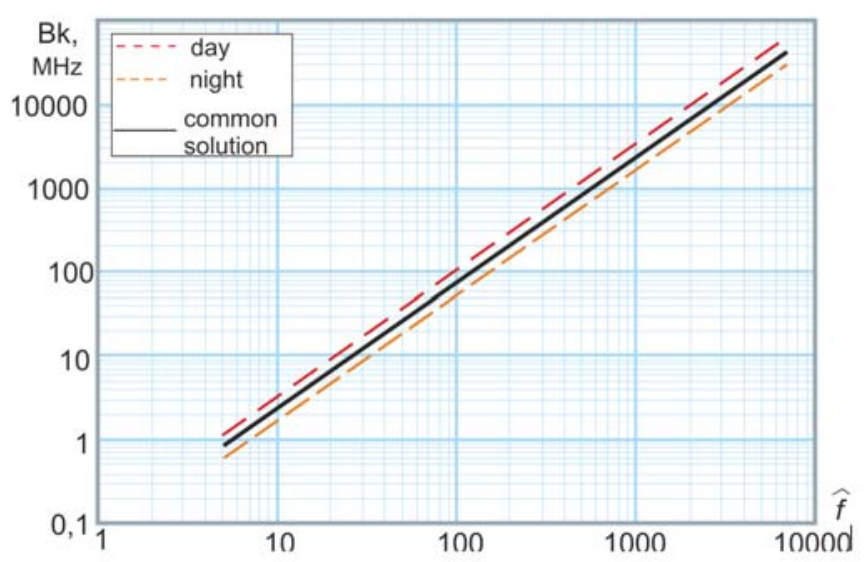

Figure 7: Coherence band depending on the relative frequency for different geophysical situations in a channel

Data comparison shows that, dependences of $C B$ on middle frequency differ from each other by no more than $20 \%$. It means that dependences of CB on relative frequency have common features.

\section{CREATION DIAGNOSTIC MAPS OF COHERENCE BANDS FOR SOME REGIONS OF RUSSIA}

Reliable operation of broadband systems is required monitoring of the order of dispersive distortions. So it is reasonable to use regional maps of coherence band with the use of data from the receiving stations of satellite navigation systems. We developed a software for creation the maps. Algorithm (look at Figure 8) of automatic creation maps of coherence bands was realized using software Autolt.

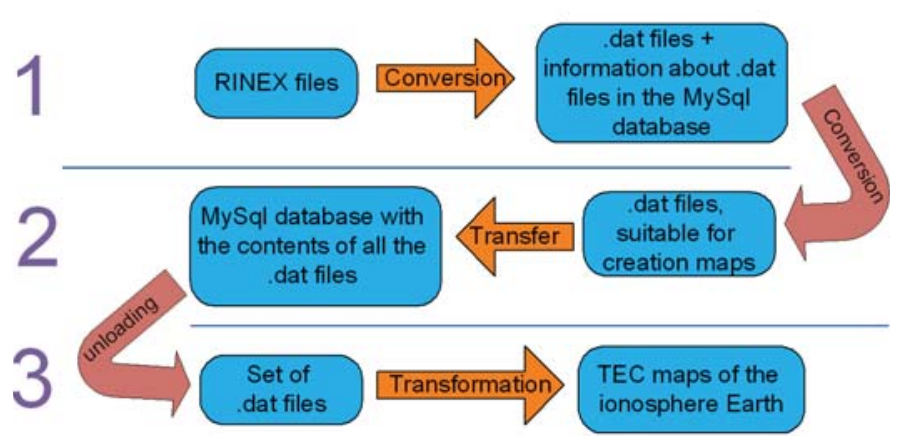

Figure 8: The algorithm of creating electronic diagnostic maps of coherence bands

It is taken into account, that the information is collected in a single database of RINEX files from multiple receivers.

The algorithm is as follows:

At first is the conversion RINEX files [11, 12] into .dat files format with the use of special software TecSuite and in the same time dat files are recorded to a database. It is done to have full information about how much and what data are available to create the electronic maps.

In the second step the existing .dat files are automatically processed using special software [6], which allows us create electronic maps of the total electron content as animated images.

The program of creation electronic diagnostic maps of the ionosphere was realized with the use of software Autolt. RINEX files are converted to .dat format using Auto It program that automates TecSuite program, and data is formed into PostgreSql database in automatic mode using Autolt language. Moreover, depending on that the file .dat already existed or has just formed in the database, the information is recorded by individual labels. This database is needed for quick access to .dat files and systematization of received information. Quick access to the files is done by special request in PostgreSql database, which work in symbiosis with the Autolt and PostgreSql, that creates a folder with the necessary dat files. This request is generated by operator, depending on the interested area and time slot. Then generated dat files are converted in the format required for creation electronic maps of coherence bands of the ionosphere in automatic mode with the use of algorithm implemented in the Autolt. Maps of coherence bands are created with the use of final information from the .dat files in automatic mode using GNU Plot programming language.

Map of coherence bands [10] is presented as animated image in .gif format, which has points that dynamically move and change colors depending on the level of total electron content (TEC), and the movement of points depends on the time and coordinate (Figure 5 ). The $\mathrm{X}$-axis represents the longitude, Y-axis is latitude, colored dots mark a place where the value of coherence band was measured. Such maps can be created for any part of the 
globe, with the appropriate data from GLONASS / GPS systems.

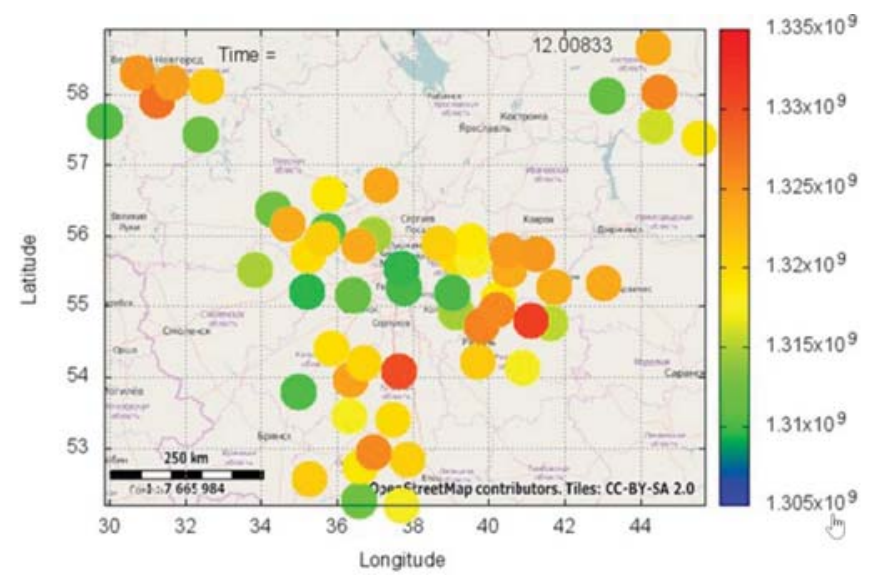

Figure 9: Regional map of coherence bands

\section{CONCLUSIONS}

Analytical expressions were obtained for the frequency dispersion parameters for various approximations of refractive index and phase shift in a channel. It is shown that dependences of dispersion characteristics on the frequency ratio (critical frequency of the ionosphere to average channel frequency) have common features. We obtained the expressions for coherence band of broadband transionospheric channels, which is an edge of undistorted frequency band of wideband signals from profile of electron concentration of the ionosphere. We developed the algorithm and software to create regional maps of coherence bands to monitor dispersive distortions and the impact on them of geophysical factors. These results will contribute to the achievement new opportunities for broadband radio systems in dispersive media, providing increased noise immunity.

This work was supported by grants № № 15-07-05280; 15-07-05294; 16-35-50083; 16-37-60068 from the Russian Foundation for Basic Research; the grants № 3.2695.2014 / K, № 8.2697.2014 / K from the Ministry of Education of the Russian Federation; the grant № 15-1910053 from Russian Scientific Foundation.

\section{REFERENCES}

1. Budden K.G. Radiowaves in the ionosphere. Cabridge: Univ. press. 1961. 542 p.

2. Cannon P. S., Angling M. J., Lundborg B. Characterization and modeling of the HF communications channel. Review of Radio Science: 1999-2002. -2002. - Pp. 597-622.

3. Gherm V. E., Zernov N. N., Lundborg B. et al.Wideband Scattering Functions for HF Ionospheric Propagation Channels. Journal of Atmospheric and Solar Terrestrial Physics. 2001. V.63. P.1489-1497.

4. Ivanov D. V., Ivanov V. A., Mikheeva N. N., Ryabova N. V., Ryabova M. I. Propagation of broadband HF signals in a medium with nonlinear dispersion. Journal of Communications Technology and Electronics. 2015. Vol. 60, No. 11. Pp. 1205-1214.

5. Ivanov V.A., Ivanov D.V., Ryabova M.I., Miheeva N.N., Katkov E.V. Broadband signal distortion in the ionosphere, caused by nonlinear frequency dispersion. Vestnik of Volga State University of Technology. Series "Radio Engineering and Infocommunication Systems". 2013. № 2(18). Pp. 5-15.

6. Kislitsin A.A. Algorithms of determination of time variations of total electron content in the upper atmosphere of the Earth. Vestnik of Volga State University of Technology. Series "Radio Engineering and Infocommunication Systems”. 2015. № 2(26). Pp. 27-40.

7. Kryukovsky A.S., Lukin D. S., Rastyagaev D. V. Research of singularities of short radio wave propagation in non-uniform anisotropic ionosphere. Electromagnetic Waves and Electronic Systems. 2009. V. 14, № 8. Pp. 17-26.

8. Kryukovskii A.S. Lukin D.S., Rastyagaev D.V., Skvortsova Y.I. Mathematical simulation of propagation of frequency-modulated radio waves in ionospheric plasma. Journal of Communications Technology and Electronics. 2015. V. 60. № 10. Pp. 1049-1057.

9. Maslin N.M. HF communications: a systems approach. London.: Pitman Publishing, 1987. Pp. 89.

10. Salous S., Bertel L.//CD-ROM Proc. Millennium Conf. on Antennas \& Propagation. (AP2000). Davos. 9-14 Mar. 2000. Noordwijk: Europ. Space Res. And Technol. Centre, 2000. P. 0958.

11. Yasyukevich Y.V., A.A. Mylnikova, V.V. Demyanov, V.V. Ivanov, N.V. Ryabova, A.A. Zuev, M.I.Ryabova, A.A. Kislitsin. Diurnal variation of vertical total electron content over the cities Irkutsk and Yoshkar-Ola according to the data of GPS/GLONASS and IRI2012 model. Vestnik of Volga State University of Technology. Series "Radio Engineering and Infocommunication Systems". 2013. № 3(19). Pp. 18-29.

12. Yasyukevich Y.V., Mylnikova A.A., Kunitsyn V.E., Padokhin A.M. Influence of GPS/GLONASS differential code biases on the determination accuracy of the absolute total electron content in the ionosphere. Geomagnetism and Aeronomy. 2015. V. 55. № 6. Pp. 763-769.

Paper submitted: 13.09.2017.

Paper accepted: 14.11.2017.

This is an open access article distributed under the CC BY-NC-ND 4.0 terms and conditions. 